\title{
GOVERNO DELL'ECONOMIA E TEORIA DELLA POLITICA ECONOMICA
}

\author{
GIUSEPPE BOGNETTI (*)
}

RIASSUNTO. - Questa relazione riguarda principalmente i due grandi protagonisti dell'illuminismo milanese (Beccaria e Verri) e si sofferma sulle loro riflessioni di carattere teorico, trascurando il ruolo da loro assunto in quanto funzionari pubblici dell'amministrazione Asburgica.

Si cerca di individuare quali sono state le coordinate concettuali che hanno consentito loro la messa a punto di indirizzi di politica economica. Ambedue vivono il clima intellettuale che guardava con ottimismo alla forza della ragione: è questa che avrebbe permesso di individuare le riforme da introdurre per migliorare la conduzione della cosa pubblica. Sostanzialmente, pur con differenziazioni, ambedue basano la loro teoria della politica economica su due considerazioni fondamentali:

(i) La prima riguarda le motivazioni dell'agire economico. Su questo argomento il dibattito nel settecento era stato vivace e aveva portato ad alcune conclusioni che gli illuministi milanesi accettano pur con una loro specificità: il comportamento egoistico del soggetto che mira al proprio interesse si può tradurre, inserito nel meccanismo del mercato, in un maggiore benessere per la società. Ne discende quindi che lo sviluppo economico richiede condizioni che lo stato o le circostanze possono certo predisporre, anche se in definitiva risulta essenziale "quel moto spontaneo della società" che non può essere creato e "modellato" da leggi direttamente restrittive.

(ii) La seconda riguarda il rapporto che si instaura tra il comando impartito dal potere pubblico e il comportamento individuale dei singoli operatori economici, siano essi produttori o consumatori. Le leggi possono addirittura ottenere risultati contrari allo scopo dichiarato per cui sono state introdotte, perché il comportamento degli agenti economici ne vanifica totalmente l'efficacia. "Gli interessi privati cospirano colla loro pluralità a deluder la legge, i custodi molteplici son sempre soggetti a inganno o a corruzione, difendere i confini esattamente colla forza fisica non si può in un sistema stabile"; o, con analogo significato, "le leggi civili devono rispettare le leggi economiche".

(*) Istituto Lombardo Accademia di Scienze e Lettere; Università degli Studi, Milano. 
All'interno di questo quadro concettuale la politica economica ha quindi principalmente il ruolo di assecondare le energie spontanee della società e di impedire che queste vengano frenate da restrizioni, da creazioni di monopoli, dall'esistenza di corporazioni. Pur all'interno di questo quadro di riferimento sostanzialmente liberale assunto da Beccaria e Verri la relazione cercherà di individuare gli strumenti di politica economica e i settori su cui intervenire da loro proposti: con particolare riguardo al pensiero del Verri, e a quanto lo differenzia dal Beccaria.

$* * *$

ABSTRACT. - Governance of the economy and theory of economic policy.

This essay explores the views on economic policy of two of the most important thinkers of the Lombard Enlightenment (Beccaria and Verri). The focus of the essay is on their theoretical contributions, so that the influence of Beccaria's and Verri's proposals on the actual course of Lombard economic reforms will not be discussed.

In Beccaria and Verri, the theory of political economy is based on a rich view of societal interdependencies and the way they operate. Beccaria and Verri developed their views within the larger context of the European economic enlightenment. Their contributions partly reflect the theories of French, English and Scottish writers. However, they were able to build on other writers' contribution in an independent and original way, often making important and influential contributions to the social theory of the European enlightenment (a standard instance is the influence Beccaria had on the development of criminal law and the formation of the utilitarian tradition).

Beccaria and Verri were convinced that reforms were possible and that the force of reason could promote solutions suitable for the improvement of society. This was not an easy task, but mainly in the first period of their intellectual activities they were hopeful to achieve important results. They were convinced that economic policy should be able to free the natural capacities of individuals so that the whole society's potential could be used to improve economic welfare. This belief comes from a very complex view of the behaviour and interaction among human beings. Society (but by no means all social conventions and institutions) was conceived as the outcome of a deliberate covenant. People agree to live together because otherwise they would live in constant insecurity for their lives and property, and because only under that covenant they would be able to enjoy a minimum degree of individual liberty.

To secure this liberty the state must respect the division of powers between the legislative, executive and judiciary branches. This model obviously derives from Montesquieu and Locke, but Beccaria and Verri develop it in an original way especially with regard the judicial power. For they believe that to give judges the power to interpret the law is to give them normative power, which would not be admissible in a system of division of powers. Therefore they believed that, in order to avoid this breach of the model, laws and statutes should be very simple and clear. In this case only, that is, only when the normative framework is certain, individuals can act in an environment of security and full liberty. From this the need derives not only to have a simple legislation 
but also a somewhat limited role of the state in managing the economy, without resorting to an extensive regulatory system. Beccaria is the one who chiefly develops this part of the model. His ideas on the judiciary exerted an important influence in the following years, starting with the French Convention (1791), in which his contributions were extensively discussed. Beccaria's views continued to attract the attention of prominent constitutional scholars (such as Laband) in the following decades and exerted a considerable influence on the drafting of a number of constitutional charts during the nineteenth century.

The overall constitutional frame, according to Verri and Beccaria, is aimed at protecting individuals in what they call 'political liberties'. What they had in mind were in fact civil liberties. Once you live under the rule of law, the state can achieve its ultimate goal that is the 'highest happiness divided by the highest possible number of individuals'. This goal is tightly connected to the belief that society is a covenant among individuals. Given this framework, how to reach this goal with an appropriate economic policy?

Three tenets underpin their proposals:

(i) The main task of economic policy is to remove obstacles to individual initiative. This is justified by the belief that the behaviour of human beings aiming at satisfying self-interest will produce the greatest welfare for society provided it is disciplined by the market process. Adam Smith expressed the same view when using the metaphor of the 'invisible hand'. But it should be noted that Verri (1768) and Beccaria (whose lectures on economia pubblica were delivered around 1770) wrote before the publication of Smith's Wealth of Nations. This theory of political economy entails that government can foster a favourable environment but cannot substitute the 'spontaneous action of society' as the latter cannot be created or 'modelled' by directly restrictive rules and statutes.

(ii) A second point concerns the relationship between direct government intervention and the results obtained. Legal norms might even produce results opposite to their explicit purpose because individuals may act in ways that hamper their effectiveness: 'Private interests with their plurality might work. against the law while authorities who should supervise the results are always subject either to deceit or corruption", or with a similar meaning "civil laws must respect economic laws". Political economy must facilitate the progress of the spontaneous energies of society, ensuring they will not be restrained by binding rules, monopolies, and guilds. Consequently, the state should act through incentives rather than through direct orders. This point of view is especially clear in Verri's treatment of the relationship between legislation and economic activity. Public finance is seen as the only field in which direct means can be used to avoid substitution effects.

(iii) Given this general approach, which one can broadly describe as liberal, Beccaria and Verri nevertheless were not dogmatic laissez faire scholars. They thought there are important reasons for the state to act. First, it is fundamental to avoid a very uneven distribution of land as this would prevent agriculture to be tolerably efficient. Moreover, government is called to 
provide vital infrastructures, public instruction, support to research to foster innovation, and so on. In other words, they were aware of what we now call market failures. Likewise, both Beccaria and Verri favoured tariffs as a sort of industrial policy, without resorting to outright prohibition of import and export. Their views were strongly criticized by laissez faire purists, but one has to agree with Schumpeter that their doctrines were only moderately protectionist and were 'a better starting point for further research than was the narrow dogmatism of the free-trade doctrine that replaced [them]'.

\section{GLI ILLUMINISTI MILANESI}

In questa lavoro faccio riferimento esclusivamente a due dei personaggi principali del gruppo degli illuministi milanesi che, riuniti attorno all'Accademia dei Pugni e alla rivista Il Caffè, hanno dato un'impronta internazionale alla vita culturale della città a partire dagli anni intorno al 1760. Mi riferisco ovviamente a Pietro Verri, che è stato il vero leader del gruppo, e a Cesare Beccaria, che ebbe con lui rapporti molto stretti fino al viaggio parigino del ' 66 , quando avvenne tra i due una rottura che non si sanò mai completamente: personaggi che hanno dato lustro alla città di Milano portandola all'attenzione del ceto culturale Europeo. Tutto ciò è confermato non solo dal notissimo riconoscimento di Schumpeter ma anche da numerosi e frequenti riferimenti, tra i quali anche un recente libro di Hutchison, ${ }^{1}$ che dedica un capitolo intero alla scuola illuministica Milanese.

Concentrerò l'attenzione sulle elaborazioni teoriche di questi due autori, utilizzando sia lavori pubblicati quando gli autori erano ancora in vita, sia lavori postumi, sia documenti da essi prodotti nel loro ruolo di alti funzionari della burocrazia asburgica; tra questi ultimi, particolarmente rilevanti le Consulte e i Voti in cui Verri espone spesso in modo sistematico e con notevole libertà il proprio pensiero su temi di grande spessore E' questo il caso delle consulte sull'Annona, sul sistema delle tariffe, sulla riforma del sistema finanziario, sulle Università delle Arti e dei Mestieri etc.Verri era uomo pronto a presentare piani molto innova-

1 T. Hutchison, Before Adam Smith, Oxford, 1988, pp. 298-307. 
tivi anche in presenza di forti opposizioni alle sue proposte. Spesso brani interi di Consulte sono stati riprodotti nei lavori più teorici, quali le Meditazioni dell'Economia, a dimostrazione del fatto che spesso la riflessione scientifica in lui scaturiva dall'osservazione dei fatti concreti.

Anche il pensiero di Beccaria, nella grande mole di documenti pubblicati nella completissima edizione nazionale, si trova spesso applicato a temi concreti e specifici: ma il suo temperamento, a differenza del Verri, non era propenso allo scontro esplicito e, quando scriveva nel ruolo di funzionario nell'amministrazione austriaca, era probabilmente condizionato dal contesto in cui operava: quindi non sempre risulta chiaro quanto questi vincoli possono aver limitato il suo vero pensiero. ${ }^{2}$ Si tenga inoltre presente che di Verri sono rimaste numerose carte private (tra le quali uno straordinario carteggio con il fratello Alessandro) scritte nel corso della sua vita fino quasi alla morte, molte delle quali non destinate alla pubblicazione immediata anche se conservate a futura memoria, che danno importanti indicazioni sull'evoluzione del suo pensiero su temi economici politici e sociologici di fondo. Per Beccaria invece non abbiamo documentazione analoga e quindi dobbiamo rifarci a quanto pubblicato in vita e postumo (tra gli scritti teorici in tema economico rimane fondamentale gli Elementi pubblicato per la prima volta dal Custodi nel 1804).

In questo scritto cerchiamo di mettere in luce soprattutto i punti di convergenza tra i due autori per descrivere il modello di politica economica che è alla base delle loro indicazioni. Non ci soffermeremo, se non in modo impressionistico, sui punti di divergenza che richiederebbero un'analisi più puntuale di quanto non sia possibile in questa sede.

Non discuteremo invece quanto essi abbiano influito sulle riforme amministrative introdotte in quei decenni nel ducato di Milano o quanto invece si siano dovuti adattare alla linea d'azione dettata da Vienna. ${ }^{3}$

\section{IL PATTO SOCIALE}

Per meglio comprendere e inquadrare il senso delle indicazioni di

2 C. Capra, Gli intellettuali e il potere: $i$ casi di Beccaria e di Verri, in L'Età dei lumi (a cura di A. Santucci), Bologna, 1998, pp. 211-258.

3 C. Capra, cit. 
politica economica dei due autori milanesi è opportuno descrivere brevemente il loro orientamento culturale e i modelli costituzionali da loro proposti; questa indagine ci consentirà di cogliere meglio il significato più profondo e la coerenza delle loro proposte.

Esaminando le coordinate concettuali che sono alla base del loro pensiero, constatiamo che ambedue appartengono al vasto movimento illuminista che ha caratterizzato il panorama culturale Europeo dell'epoca. Ambedue guardavano con ottimismo alla "forza della ragione" che doveva squarciare le tenebre dell' "epoca oscura" e illuminare, almeno in linea di principio, la strada da percorrere per migliorare e rendere più civile la convivenza comune. Sostanzialmente, pur con differenti accentuazioni, ambedue poggiano la loro teoria della politica economica su una visione comune circa il modo di operare della società e le finalità da perseguire.

La finalità dell'azione di governo è, per Beccaria, bene espressa dalla frase: "La massima felicità divisa nel maggior numero" ${ }^{4}$ e per Verri "(la) felicità pubblica o sia la maggior felicità possibile divisa colla maggior uguaglianza possibile". ${ }^{5}$ Una locuzione, questa, che troviamo in un certo numero di autori appartenenti agli ambienti intellettuali del settecento e, per quanto se ne sa, utilizzata per primo da Hutcheson. ${ }^{6} \mathrm{Nei}$ nostri due autori essa assume da un lato, specie per Beccaria, una piega utilitarista molto forte, dall'altro indica che la felicità è riferita esclusivamente ad una condizione psicologica individuale. In questo sono quindi ambedue contrari all'idea di Rousseau che l'uomo è fatto dalla società e per la società?

Per raggiungere l'obiettivo della felicità pubblica è necessario preliminarmente che si formi una società con i relativi diritti. Perché questo avvenga deve nascere un patto che leghi tra di loro gli individui: per Beccaria il patto nasce perché gli uomini “.. stanchi di vivere in un continuo stato di guerra e di godere di una libertà resa inutile dall'incer-

4 C. Beccaria, Edizione Nazionale, Vol. I, Dei delitti e delle pene, p. 23.

5 P. Verri, Meditazioni sulla felicità, p. 84.

6 Secondo Francioni è difficile che Beccaria si sia rifatto direttamente a Hutcheson, An inquiry into the original of our Ideas of Beauty and Virtue. Un altro autore che usa questa espressione è Helvétius. Si veda C. Beccaria, Dei delitti, cit., p. 23 , nota 1 .

7 Su Rousseau si veda G. Tarello, Storia della cultura giuridica moderna, Bologna, 1976, p. 320 e sgg. 
tezza di conservarla.. ne sacrificarono una parte per goderne il restante con sicurezza e continuità".

E' chiaro per Beccaria che la limitazione alla libertà individuale è circoscritta, in quanto funzionale al massimo grado di libertà conseguibile quando si vive in società. Ma quali sono i diritti che nascono dalla conclusione del patto sociale? $\mathrm{E}$ in particolare quali basi ha il diritto di proprietà? Su questo punto i due hanno un atteggiamento parzialmente differente, che tuttavia non sembra essere stato tale da portarli a conclusioni molto diverse sui temi di politica economica. Beccaria ha un rapporto tormentato con il diritto di proprietà che, come è noto, egli definisce, nella terza stesura Dei delitti e delle pene, "un diritto terribile forse non necessario". ' Il diritto di proprietà è un diritto che serve a facilitare e rendere possibile il contratto sociale; infatti senza il riconoscimento del diritto di proprietà l'individuo non sarebbe disposto a rinunciare alla propria libertà di azione. Tuttavia questo diritto non appartiene ad una sfera intoccabile; non è un diritto irrinunciabile come lo è ad esempio in Locke. Negli Elementi egli ribadisce questa sua posizione "Mi si obietterà di primo slancio: qual giustizia d'impedire a ciascheduno di trarre a suo arbitrio quel profitto ch'egli voglia dai propri fondi? ...Si deve ciò non ostante considerare che la proprietà è figlia primogenita e non madre della società... Da ciò si vede chiaramente essere la proprietà soggetta alle leggi....". ${ }^{10}$ Anche per Verri il diritto di proprietà ha natura pattizia ma egli lo considera un punto fermo che non viene mai da lui posto in discussione: "pour peu qu' on raisonne sur les lois de nature on trouvera qu'il n'y en a d'autre que la force" ${ }^{11} \mathrm{E}$ perciò "Avant qu'il y ait une Costitution il n'y a point de sureté, point de proprieté. Point de liberté, point de droit" ${ }^{12}$ Inoltre quello che Verri sottolinea è che solo una buona costituzione, presumibilmente ispirata a principi illuministici, garantisce appunto i diritti di proprietà, di sicurezza etc. Comunque Verri si discosta dalla presa di posizione dell'autore Dei delitti e delle pene. Nel 1766, quando già è in rotta con Beccaria, critica l'espressione che compare nei Dei delitti e delle pene
8 C. Beccaria, Dei delitti, cit., p. 11.
9 C. Beccaria, Dei delitti, cit., p. 75.
10 C. Beccaria, Elementi, $11^{\circ}$ vol., cit., p. 229.
11 P. Verri, Idées sur la societé, in Edizione Nazionale, Vol. VI, p. 519.
12 P. Verri, Della dichiarazione dei diritti, in Edizione, Vol. VI, p. 625. 
sia per quello che riguarda il termine "terribile" sia soprattutto per quel "non necessario" che ritiene del tutto sbagliato e solo accettabile in una società completamente totalitaria: "On dit que dans une province de l'Amerique Meridionale il n'y ait pas cet droit elle est ce pendant peuplée et cultivée; d'accord mais par des esclaves qui vivent sou le dispotisme le plus absolu". ${ }^{13}$

Per i nostri autori comunque, contrariamente a quanto afferma Locke, la proprietà non è un diritto naturale che preesista al patto sociale ma deriva dagli accordi pattizi. Tuttavia per Verri il diritto di proprietà, pur essendo di natura pattizia, ha comunque un grande rilievo nei rapporti sociali ed è imprescindibile per un buon funzionamento della società.

\section{LA RIPARTIZIONE DEI POTERI}

\section{Il potere legislativo}

Per ottenere la maggiore felicità per il maggior numero di persone, secondo i nostri autori, il potere statuale deve essere diviso: in particolare bisogna tenere distinti il potere legislativo da quello giudiziario. Questa divisione si ispira alle idee di Montesquieu, come è espressamente detto da Beccaria nell'Introduzione a Dei delitti e delle pene e a quelle di Locke; l'attenzione dei due milanesi però si sofferma soprattutto su due poteri, il legislativo e il giudiziario.

Innanzitutto bisogna avere un sistema legislativo che ponga le norme in termini generali; esse rappresentano il fulcro dell'intero sistema in quanto servono da riferimento all'attività di ciascun cittadino senza distinzione alcuna. ${ }^{14}$

Nel sistema costituzionale dei nostri due autori preminente è il

13 P. Verri, Osservazioni sul trattato Dei delitti e delle pene di Cesare Beccaria, in P. Verri, Osservazioni sulla tortura (a cura di G. Barbarisi), Milano 1985, p. 161. Questa divergenza tra Beccaria e Verri richiama alla mente quella tra Einaudi e Croce su liberismo e liberalismo.

14 Sull'importanza che riveste la legge nel sistema di Verri si veda P. Porta, R. Scazzieri, Il contributo di Pietro Verri alla teoria economica, in C. Capra (a cura di), Pietro Verri e il suo tempo, Bologna, 1999, Tomo II, pp. 813-846. 
ruolo del legislatore il quale deve provvedere a promulgare leggi "giuste" a vantaggio della comunità. E' evidente che negli scritti a cui facciamo riferimento, stesi negli anni sessanta, il soggetto titolare della funzione normativa era ovviamente il sovrano illuminato, il cui interesse si riteneva dovesse coincidere con quello della comunità in quanto appunto nel proprio interesse avrebbe governato meglio con il consenso dei propri sudditi: "Qualunque sia la forma di governo... sarà sempre vero che la giustizia e l'interesse del sovrano esigono di lasciare ai cittadini la maggiore libertà possibile, e togliere loro quella sola porzione di naturale indipendenza che è necessaria a mantenere l'attuale forma di governo". ${ }^{15}$ Una presa di posizione diversa non era in quella fase storica possibile, se non mettendosi in una posizione rivoluzionaria totalmente al di fuori dal sistema. Per temperamento sia Beccaria sia Verri erano invece saldamente riformisti e sempre lo saranno nel corso della loro vita pubblica. Verri tuttavia, al di là delle condizioni contingenti, quando scrive le Meditazioni credeva sinceramente nel dispotismo illuminato, convinto com'era che il contrasto di interessi dei gruppi di potere esistenti nella società avrebbero impedito l'introduzione di una riforma coerente ritenuta invece necessaria e possibile solo con l'intervento del sovrano illuminato. Nelle Meditazioni Verri propone che un dittatore, simile nelle sue caratteristiche al dittatore romano e cioè a tempo, intervenga per introdurre nuove regole che rispettino gli interessi generali della comunità. "Quando si tratta di decidere i casi particolari a norma delle leggi già pubblicate, la diversità delle opinioni umane rende appunto difficile l'ingiustizia, perché l'una contempera l'altra; ma quando si tratta d'agire, e di un'azione pronta, spedita e sempre uniforme ad un fine, io non credo potersi ciò far dipendere dalla pluralità dei voti”. ${ }^{16}$

La preferenza di Verri per l'assolutismo illuminato, anche se con diversi gradi di intensità, permane fino a quando Giuseppe II sale al trono. Con l'avvento del nuovo sovrano Verri viene elevato alla presidenza della Magistratura Camerale (sebbene da questo organo sia scorporata la competenza sulle materie finanziarie); in questa occa-

15 P. Verri, Sulle leggi vincolanti principalmente nel commercio de' grani, in Edizione, cit., Vol. II, Tomo II, p. 248.

16 P. Verri, Meditazioni sull'economia politica, in Edizione, cit., Vol. II, Tomo II, p. 556. 
sione esprime ancora al fratello Alessandro il proprio entusiasmo per la soluzione che si stava prospettando con un sovrano illuminato deciso a introdurre le riforme necessarie. ${ }^{17}$ Verri cambierà poi parere anche sulla spinta del suo brutale allontanamento dall'amministrazione e i suoi giudizi su Giuseppe II diventeranno molto severi. I ripensamenti saranno profondi con il sopraggiungere della rivoluzione francese.

\section{Il potere giudiziario}

Prima di descrivere l'evoluzione del pensiero di Verri sull'assetto che il potere legislativo dovrebbe assumere vorremmo richiamare l'attenzione sull'importanza che i due annettono al corretto funzionamento del potere giudiziario.

Le idee di Beccaria e Verri sul potere giudiziario sono chiaramente ispirate da Montesquieu (soprattutto per il rapporto tra legislativo e giudiziario) e da Locke (soprattutto per il rapporto tra esecutivo e giudiziario). ${ }^{18}$ Essi sono maggiormente interessati al primo rapporto, e infatti è su questo che si soffermano e sviluppano le loro argomentazioni con grande determinazione e rigore intellettuale.

Per tutti e due la divisione tra i due poteri deve essere netta; soprattutto per loro è chiara la ragione per cui deve esserci separazione: si vuole evitare che il giudice diventi esso stesso legislatore.

Beccaria infatti scrive nel Dei Delitti: "nemmeno l'autorità d'interpretare le leggi penali può risiedere presso i giudici criminali per la stessa ragione che non sono legislatori" ${ }^{19}$ Aggiunge anche: "in ogni giudizio si deve fare dal giudice un sillogismo perfetto: la maggiore dev'essere la legge generale, la minore l'azione conforme o no alla legge, la conseguenza la libertà o la pena" ${ }^{20} \mathrm{E}$ analogamente Verri afferma: "Nel momento in cui il giudice applica una sua interpretazione crea in realtà una nuova norma"... "Interpretare significa far

17 P.A. Verri, Carteggio, Vol. XI p. 228.

18 Ch. Montesquieu De l'esprit des lois, cap.VI, 3 e cap. XI, 6; J. Locke, Of Civil Government, cap. VIII e X.

19 C. Beccaria, Edizione Nazionale, Dei delitti e delle pene, in Edizione, Vol. I, p. 36.

20 C. Beccaria, Dei delitti, cit., p. 36. 
dire al legislatore più di quello che ha detto e quel più è la misura del potere legislativo che si arroga il giudice" ${ }^{21}$

L'importanza e l'influenza della argomentazioni degli illuministi milanesi a favore della decisa separazione del potere legislativo da quello giudiziario è testimoniata dalla discussione tenuta all'Assemblée Nationale nel 1790 su argomenti giurisdizionali: le tesi di Beccaria, soprattutto nella formula del sillogismo, furono spesso al centro del dibattito. $^{22}$ Certamente Montesquieu (e Locke) hanno anticipato sia Verri che Beccaria sul ruolo del potere giudiziario, ma il pensiero dei due milanesi, soprattutto attraverso la formulazione del sillogismo di Beccaria, ha avuto un suo sviluppo autonomo e ha costituito un punto di riferimento per le discussioni a venire non solo, come detto, nel dibattito delle costituenti francesi del periodo rivoluzionario ma anche in quello che ha accompagnato in vario modo il modelle di costituzione liberale dell' $800 .^{23}$

Secondo Verri e Beccaria quando il giudice può interpretare la legge si crea un problema di legittimazione, in quanto solo il sovrano è il titolare del processo legislativo. La legge non ha più quel carattere generale richiesto per un corretto vivere civile quando l'interpretazione

21 P. Verri, Sulla interpretazione delle leggi, in Il Caffè (a cura di G.Francioni, S. Romagnoli), Torino, 1993, p. 698. Lidea che l'interpretazione equivale a un atto normativo risale addirittura a Bacone. Infatti tra gli aforismi che Beccaria aveva trascritto dalle opere di Bacone vi sono i seguenti: "Cum receditur a littera, iudex transit in legislatorem"; "Non est interpretatio, sed devinatio, quae recedit a littera". Su questo punto si veda Dei Delitti, cit., nota 2 a pag. 37. Anche Verri cita Bacone nel Sull'interpretazione, cit., "Si iudex transige in legislatorem, omnia in arbitrium penderent", p. 698.

22 Su questo si veda Y. Hufteau, Le référé législatif, Paris, 1965, p. 38, nota 1: "l'analyse du jugement comme syllogisme.. Duport et Garat l'ainé l'empruntèrent à Beccaria”. Duport e Garat l'ainé sono due rappresentanti che discussero a lungo il tema gurisdizionale nell'Assemblée Nationale.

23 Sull'influenza esecitata da Beccaria sulle costituzioni del periodo rivoluzionario si veda anche F. Di Donato La costituzione fuori dal tempo, in Quaderni Costituzionali, 2011/4, pp. 895-926. In particolare "..il permanente clima di diffidenza e di osti lità per la giurisdizione derivato dall'esperienza dei parlamenti di Antico Regime ispirò nei testi costituzionali francesi una concezione molto restrittiva della funzione giudiziaria che assorbiva gli orientamenti dottrinali illuministici soprattutto di Montesquieu e di Beccaria”, p. 920. Per l'influenza del sillogismo di Beccaria si veda anche Y. Hufteau, cit., p. 38, che ricorda che Anton Laband, insigne giurista tedesco lo utilizzava ancora in pieno Ottocento. 
del giudice è atto individuale che spesso non può essere previsto. Viene quindi meno la certezza del diritto e, secondo Verri, viene meno quella che egli definisce la libertà politica: “..col nome di libertà politica io intendo l'opinione che ha ogni cittadino di possedere se medesimo e quello che è suo e di poterne a suo piacere disporre sin tanto ch'ei non trasgredisca le leggi promulgate con legittima autorità". ${ }^{24}$

E' importante rilevare le implicazioni delle tesi di Verri e di Beccaria circa il modo corretto di legiferare: bisogna governare attraverso leggi semplici per renderne più agevole l'applicazione. Ancora più importante ai fini della politica economica è il rilievo che rispettare la separazione dei poteri significa limitare di fatto anche la dimensione dell'intervento dello stato. Gli interventi del soggetto pubblico nelle attività private devono quindi essere quantitativamente limitate in quanto uno stato molto interventista necessariamente deve predisporre norme molto circostanziate e complicate, richiedendo quindi al giudice un'attività interpretativa molto penetrante e facendo quindi venir meno la certezza del diritto. Le affermazioni dei due milanesi alle nostre orecchie hanno un suono estremistico, tanto che Sergio Romagnoli, nel convegno dedicato al $250^{\circ}$ centenario della nascita di Beccaria, dichiara: " $E$ ' curioso che proprio in questo capitolo (quello sull'interpretazione contenuto nei Dei delitti e delle pene) si debba avvertire la maggior distanza tra noi e il pensiero giuridico illuministico giacché oggi non possiamo condividere l'assunto che lo governa in quanto riconosciamo unanimemente il diritto del giudice di interpretare le leggi penali" ${ }^{25}$ Nella situazione attuale lo stato è molto presente a vario titolo nell'economia (e non solo in quella) e intermedia una quota del Pil che nei paesi avanzati si colloca tra il quaranta e il cinquanta percento; la posizione dei due illuministi milanesi suona quindi anacronistica poiché è difficile immaginare una società organizzata come la nostra nella quale il giudice non eserciti un'attività interpretativa. Tuttavia le tesi dei nostri due autori sono più plausibili se intese come un orientamento a limitare alquanto le libertà interpretative del giudice in modo di garantire al massimo la certezza del diritto: questo fu certamente uno degli obiettivi al centro del dibattito sui modelli costituzionali dell'ottocento. Il con-

24 P. Verri, Sulla interpretazione, cit., p. 600.

25 S. Romagnoli, Prolusione, in Convegno su Cesare Beccaria tra Milano e l'Europa, Milano, 1990, p. 6. 
tributo (specie quello di Beccaria) a questa discussione non fu irrilevante. ${ }^{26}$

Il pericolo che la forte discrezionalità nell'interpretazione delle leggi e la proliferazione delle stesse potesse intralciare le attività economiche era del resto già presente in Verri nelle Considerazioni sul Commercio, ${ }^{27}$ e ribadito nelle Memorie sull'economia dello Stato di Milano. Nelle Considerazioni scrive: "La speranza d'ogni privato d'arricchirsi è quella che anima l'industria, la quale ben diretta forma l'utile commercio, ma la speranza d'arricchire suppone la sicurezza a ciascuno di quello che è suo. Questa sicurezza non può aversi se non quando le possessioni private venghino regolate nel codice delle leggi colla maggior possibile chiarezza e precisione, di modo che al giudice o al tribunale qualunque di giustizia altro non spetti che il decidere se il caso controverso sia quello della legge" ${ }^{28}$ Anche nello scritto più tardo (a metà degli anni 80) sul sistema giudiziario ribadisce la necessità di limitare la libertà di interpretazione del giudice ricorrendo a leggi molto semplici. ${ }^{29} \mathrm{E}^{\prime}$ chiaro anche che i nostri due autori non erano contrari al coevo movimento di codificazione; Verri del resto, nell'articolo Sull'interpretazione delle leggi, afferma l'opportunità di adottare un meccanismo che tipizzi le fattispecie normative rendendole chiare e semplici. L'auspicio è quindi avere poche e chiare norme che possono essere applicate direttamente senza un'attività interpretativa del giudice. Ne deriva anche che lo stato deve essere poco interventista, in sintonia con il modello liberale.

\section{IL COSTITUZIONALISMO DEL TARDO VERRI}

Rimane da vedere come Pietro Verri abbia modificato il proprio

26 Per una descrizione della divisione dei poteri dello stato nel modello liberale e del perché questa sia stata superata nei nostri ordinamenti orientati verso il modello di stato sociale o del benessere si veda Giovanni Bognetti, La divisione dei poteri, Milano, 1994, specialmente capitoli III, IV, V.

27 P. Verri, Considerazioni sul Commercio, in Edizione, cit., Vol. II, tomo I, pp. 107-296; P. Verri, Memorie sull'economia pubblica dello Stato di Milano, in Edizione, cit., Vol. II, tomo I, pp. 348-435.

28 P. Verri, Considerazioni, cit., p. 228.

29 P. Verri, Idee sul sistema giudiziario, in Edizione, cit., Vol. VI, pp. 313-321. 
giudizio sul tipo di governo da preferire.$^{30} \mathrm{La}$ rivoluzione francese gli ha fatto percepire che era possibile in Europa, se si esclude l'Inghilterra già retta una monarchia costituzionale, introdurre un sistema di governo diverso da quello esistente negli stati dell'Ancien Règime. Al contrario del fratello Alessandro il giudizio sugli eventi francesi è positivo e, pur condannando gli eccessi sanguinari e l'uccisione del re, li giustifica almeno in parte, sostenendo che quando vi sono rotture nell'evoluzione dei processi storici difficilmente si riescono ad evitare atti violenti. Va tuttavia ricordato che Verri già inizialmente esprime delle riserve sulla possibilità di applicare in modo semplice il caso francese a quello italiano; riserve dovute ai suoi forti dubbi sulla maturità degli Italiani, che di fronte ad eventi analoghi a quelli francesi avrebbero potuto reagire in modo tale da portare il paese al più totale disordine. ${ }^{31}$

L'influenza dei fatti francesi si ritrova già in una sua nota del 1790 Pensieri sullo stato politico del Milanese nel 1790, sollecitata dalla richiesta di Leopoldo che chiedeva suggerimenti in merito alla costituzione che avrebbe dovuto reggere lo stato di Milano. Lo scritto ci sembra molto importante perché chiarisce i cambiamenti del pensiero di Verri sul sistema costituzionale da adottare mostrando quanto vi è di continuità rispetto al periodo precedente e quanto invece è cambiato. Il sistema previsto da Verri ruota tutto intorno alla proprietà: "Una costituzione finalmente conviene cercare, cioè una legge inviolabile anche ne' tempi a venire, la quale assicuri ai successori la fedeltà nostra de buoni e leali sudditi e assicuri ai nostri cittadini una inviolabile proprietà, essendo questo il fine ultimo di ogni governo". ${ }^{32}$ Similmente nel paragrafo successivo, intitolato il Cardine di tutto, la frase iniziale è

30 Su questi temi come del resto su tutto il pensiero di Verri fondamentale sono il lavori di C. Capra, I progressi della ragione, Bologna, 2002 e le note introduttive al VI volume dell'edizione nazionale delle opere di P. Verri, Scritti politici della maturità. Sullo scambio di giudizi sulla rivoluzione francese tra Pietro e Alessandro si veda: Pietro e Alessandro Verri, Carteggio, in Edizione, cit., Vol. VIII, due Tomi.

31 "cosa accaderà dell'Italia? Siamo immatutri e non ancora degni di vivere sotto il regno della virtù.. Se non si illumina prima la plebe, s'ella non costringe poi i nobili a piegarsi, una rivoluzione da noi non può cagionare che rapine e saccheggi, rinnovando le sciagure de' guelfi e ghibellini”. P. Verri, Alcuni pensieri sulla rivoluzione francese, in Edizione nazionale, cit., Vol. VI, p. 471.

32 P. Verri, Pensieri politici del Conte Pietro Verri sullo Stato politico del Milanese nel 1790, in Edizione, cit., Vol. VI, p. 408. 
Sicurezza della proprietà (i corsivi sono dell'autore). Con questa espressione Verri intende: "ogni uomo sia in avvenire sicuro sotto la protezione della legge e nella persona e ne beni... la libertà personale deve essere garantita sin tanto che non si sia violata una legge".3.

La sicurezza della proprietà riguarda anche il tributo sulla terra che deve essere stabile nel tempo (approva quindi Carlo VI e Maria Teresa che avevano assicurato che il carico sulle terre sarebbe rimasto inalterabile). Parimente "la sicurezza della proprietà viene tolta colle arbitrarie addizioni di aggravio della tariffa de' dazj" ${ }^{34}$ Le imposte possono essere variate solo con l'assenso dello Stato; la delibera deve avvenire dopo la consultazione del Corpo rappresentante lo Stato, composto da rappresentanti eletti periodicamente su base censuale. Viene introdotto quindi il principio del no taxation without representation, anche se solo in via consultiva. Verri abbraccia ora dottrine che sempre più si avvicinano a quelle di Locke, che sosteneva che la potestà legislativa trovava il suo fondamento nella volontà di un corpo elettorale; nel periodo successivo si parla di potere legislativo conferito ad una assemblea nazionale i cui eletti siano "scelti dai distretti a liberi voti degli abitanti". ${ }^{35}$ Dopo l'invasione dei francesi del '96, partecipando alla vita politica Verri prenderà posizione contro i giacobini milanesi esprimendo la propria preferenza per una soluzione moderata. ${ }^{36}$ L'ultimo articolo che scrive nel 1797 per il giornale milanese progressista è improntato a un atteggiamento paternalistico che indica la preoccupazione che il nuovo assetto istituzionale non conduca ad un sovvertimento del sistema economico. ${ }^{37}$

33 P. Verri, Pensieri, cit., p. 409.

34 P. Verri, ibidem, p. 414.

35 P. Verri, Primi elementi per somministrare al popolo delle nozioni tendenti alla felicità, in Edizione, cit., vol. VI, p. 646. Secondo Capra qui Verri non pensa tanto ad al suffragio universale ma al sistema elettorale previsto dalla costituzione francese del 1791 che distingueva tra cittadinanza attiva e passiva Nota introduttiva, in Edizione, cit., vol. VI, p. 605.

36 Sul moderatismo di Verri si veda il saggio di C. Dipper, Dispotismo e costituzione: due concetti di libertà, in Economia, istituzioni, cultura in Lombardia nell'età di Maria Teresa, (a cura di A. De Maddalena, E. Rotelli, G. Barbarisi, Bologna, 1982, pp. 863-902.

37 P. Verri, Pensieri di un buon vecchio che non è letterato, in Edizione, cit., vol. VI, pp. 808-815. 
$\mathrm{Ci}$ si può chiedere a questo punto se le nuove idee abbiano cambiato considerevolmente quanto aveva sostenuto fin dai primi anni Verri in tema di libertà civili e di libertà economiche. Ci pare che questo non sia avvenuto e che sull'attività economiche il suo pensiero abbia mantenuto una linea costante. Se quindi per l'aspetto relativo alle libertà politiche Verri ha cambiamenti anche di notevoli entità, per quello invece che riguarda le libertà civili non sembra avere dubbi e rimane fermo sulle sue posizioni che saranno al centro dell'evoluzione del pensiero liberale del diciannovesimo secolo. Centrale è la libertà nell'attività economica e fondamentale per questa è la protezione della proprietà privata. Negli ultimi saggi le idee di Verri sulle libertà civili sono analoghe a quelle che si trovano nel saggio Sull'interpretazione delle leggi (dove come abbiamo detto venivano chiamate libertà politiche).

\section{FONDAMENTI DELL'AGIRE ECONOMICO E GLI STRUMENTI DELLA POLITICA ECONOMICA}

All'interno di questo quadro istituzionale e coerentemente con esso Verri e Beccaria elaborano il modello di politica economica da seguire. Esistono tra i due notevoli consonanze che non escludono però divergenze di una certa importanza: in generale Beccaria mostra di essere molto più cauto nello spingere l'indirizzo liberista, seguito da entrambi, fino all'estreme conseguenze.

Nel quadro costituzionale sopra delineato come si inserisce la politica economica? Due sono le caratteristiche dell'agire dell'uomo in società che vengono riconosciute essere alla base dei comportamenti degli agenti economici e di governo.

La prima riguarda le motivazioni dell'agire individuale nel campo economico, argomento sul quale il dibattito nel Settecento era stato vivace. Il punto di partenza di Verri e di Beccaria non si discosta molto da una letteratura che trae origine dalle considerazioni di Mandeville, esposte nel Dialogo delle api $i^{38}$ su come vizi privati potessero trasformarsi in pubbliche virtù. Il comportamento egoistico del soggetto che mira al proprio interesse si può tradurre, inserito nel meccanismo del

38 B. Mandeville, The Grumbling Hive, London, 1705. 
mercato, in un maggiore benessere per la società: "Travaglia egli per il bene della società quando vi trova l'utile proprio. La grand'arte del legislatore è di sapere ben dirigere la cupidigia degli uomini. Allora si scuote l'utile industria dei cittadini; l'esempio, l'emulazione e l'uso fanno moltiplicare i cittadini utili, i quali cercano a gara di farsi più ricchi col somministrare alla patria merci migliori a minor prezzo.... laonde l'arte di scrivere buone leggi si è appunto di far coincidere l'interesse privato col pubblico". ${ }^{99}$ Verri ribadisce ancora nelle Leggi vincolanti: "L'interesse privato di ognuno, quando coincide col pubblico interesse, è sempre il più garante della felicità pubblica"..$^{40}$

La somiglianza di queste frasi con quelle famose usate da Adam Smith nella Ricchezza delle Nazioni "it is not from the benevolence of the butcher, the brewer, or the baker, that we expect our dinner, but from their regard to their own interest" è evidente, e notevole è il fatto che Verri anticipa di dieci anni Adam Smith. ${ }^{41}$

Analogamente Beccaria ribadisce nei Dei delitti e delle pene "Nessun uomo ha fatto il dono gratuito di parte della propria libertà in vista del ben pubblico; questa chimera non esiste che ne' romanzi..." ${ }^{42}$ $\mathrm{Ne}$ discende quindi che lo sviluppo economico richiede condizioni che lo stato o le circostanze possono certo predisporre, anche se in definitiva risulta essenziale "quel moto spontaneo della società" ${ }^{43}$ che non può essere creato e "modellato" da leggi direttamente positive o restrittive.

La seconda importante considerazione riguarda il rapporto che si instaura tra il comando impartito dal potere pubblico e il comportamento individuale dei singoli operatori economici, siano essi produttori o consumatori. Le leggi, nella loro visione, possono addirittura ottenere risultati contrari allo scopo dichiarato per cui sono state introdotte perché il comportamento degli agenti economici può vanificarne totalmente l'efficacia. Troviamo ad esempio espressioni del tipo "Gli

39 P. Verri, Sulle leggi, cit., p. 249.

40 P. Verri, Sulle leggi, cit., p. 43.

41 Si tenga presente che Smith aveva nella sua biblioteca le Meditazioni di Verri e che sapeva leggere l'italiano; si potrebbe perciò persino pensare che i concetti espressi da Verri abbiano esercitato una qualche influenza sull'economista scozzese. Su questo e sull'interazioni tra Smith e Verri si veda P. Porta, Nota Introduttiva, in Edizione, cit., Vol. I, tomo II, specialmente p. 55 e sgg.

42 C. Beccaria, Dei delitti, cit., p. 30.

43 P. Verri, Meditazioni, cit., p. 427. 
interessi privati cospirano colla loro pluralità a deluder la legge, i custodi molteplici son sempre soggetti a inganno o a corruzione"; ${ }^{44} \mathrm{o}$, con analogo significato, "Facciasi una legge conforme alla verità, e cesserà la disobbedienza del popolo, o per meglio dire l'errore della legge". ${ }^{45}$

I limiti degli interventi del potere pubblico dipendono innanzi tutto dal fatto che è necessaria l'adesione spontanea della maggioranza degli agenti economici perché non sia vanificato l'obiettivo che si vuole raggiungere; quindi difficilmente questo può essere totalmente contrario all'interesse individuale dei singoli componenti della collettività. In secondo luogo si nutre un notevole scetticismo sulle capacità di controllo, vuoi per carenze conoscitive vuoi per limiti istituzionali della pubblica amministrazione. E' possibile pertanto che l'intervento pubblico, producendo effetti non previsti, sia dannoso al punto da frenare o impedire del tutto lo sviluppo economico. Tale era stato quello del governo spagnolo che, esercitando forti interferenze sull'attività economica, togliendo il diritto speciale alle attività commerciali e oberando le attività economiche con imposte troppo elevate e distribuite "viziosamente" e mal collocate, aveva contribuito con ciò alla decadenza economica di Milano. ${ }^{46}$ Queste considerazioni portano Verri ad elaborare una vera e propria teoria generale della politica economica che si concretizza in due massime. La prima viene formulata in questi termini: "le leggi di finanza se sono indirette sono pessime"; la seconda invece afferma che le leggi di economia pubblica sono per lo contrario pessime se sono dirette". ${ }^{47}$ Assegna anche due specifici compiti alla politica tributaria e alla politica economica: "la finanza ha per oggetto di legar meno che si può la nazione nel ripartimento del tributo; l'economia pubblica ha per oggetto di accrescere al maggior grado possibile l'annua riproduzione" 48 (quando Verri usa l'espressione economia pubblica intende parlare del sistema economico nel suo complesso e non fa riferimento

44 P. Verri, Sulle Leggi vincolanti, cit., p. 256.

45 C. Beccaria, Del disordine e de' rimedi delle monete nello stato di Milano nell'anno 1962, in C. Beccaria, Opere Vol. XII (Collana Custodi, Milano, 1804), p. 325.

46 P. Verri, Memorie sulla economia pubblica dello Stato di Milano, in Edizione, cit., Vol. III, Tomo I, pp. 373, 376, 392.

47 P. Verri, Meditazioni sull'Economia Politica, in Scritti di economia, finanza e amministrazione, Edizione Nazionale delle Opere di Pietro Verri, Vol. II, Tomo 2, Roma, 2007, p. 552.

48 P. Verri, Meditazioni, cit., pp. 551. 
al settore pubblico dell'economia). Che cosa intende per intervento diretto viene spiegato da Verri con qualche esempio: "se si vuole accrescere la popolazione o far aumentare la superficie coltivata o aumentare il livello qualitativo dei prodotti non serve proibire l'emigrazione, obbligare ogni cittadino a sposarsi prima di una certa età, comandare di mettere a cultura tutte le terre; questi comandi porterebbero nella popolazione un clima di disordine e di desolazione. Il provvido ministro di economia colle preferenze ed onori renderà rispettabile lo stato coniugale; rianimerà l'industria col toglierli i ceppi, collo spianarvi le strade, coll'assodare la proprietà, preziosissimo bene dell'uomo sociale, col procurare agli abitanti un'intima persuasione della sicurezza propria, nel che solo consiste la libertà civile" ${ }^{49}$

L'intervento dello stato inoltre può avere, per le ragioni più sopra ricordate, efficacia limitata: "Il ministro vede che nella politica più giova il lasciar fare che il fare: che la somma delle azioni d'una società ha per elementi tutte le minime sensazioni d'ogni uomo inconoscibili e incalcolabili: che il Pirronismo e la cautela debbono precedere ogni operazione sulla società: che l'industria degli uomini né si eccita, né si frena, ma si scioglie e si dirige utilmente: che l'avidità personale di ognuno, lasciata in libertà, è sempre il mezzo più attivo e costante per rintuzzare l'avidità personale di ognuno". ${ }^{50}$ In sostanza Verri, in accordo con Beccaria, ritiene che bisogna in generale ricorrere a strumenti incentivanti e quindi non a norme o regolarmente rigidi che impongono divieti assoluti.

I principi che debbono muovere il ministro di finanza sono invece in gran parte diversi dai principi che debbono muovere un ministro di economia pubblica. Dice Verri: "le leggi di finanza se sono indirette sono pessime.... Se nella finanza vorrà percepirsi un tributo per legge indiretta, per esempio, proibire a tutti i cittadini un'azione, non già perché realmente essa si voglia impedire, ma affinché comprino la dispensa per farla (delle quali leggi in molti paesi ve ne sono) dico che questo tributo indiretto costerà alla nazione assai più di quello che ne ricava l'erario, e importerà molte volte la venalità, la corruzione e una dispersione di tempo in uffizi. Laonde se chiaramente e direttamente

49 P. Verri, Meditazioni sull'economia politica, in Edizione, cit., Vol. II, Tomo 2, Roma, 2007, p. 552.

50 P. Verri, Sulle leggi vincolanti, cit., p. 260. 
le leggi di finanza ordinasse il pagamento d'una somma corrispondente sul fondo censibile, sarebbe assai più naturalmente e placidamente collocato il tributo" ${ }^{51}$ L'imposta ha un duplice effetto: diminuisce il reddito e sposta la convenienza tra diverse azioni facendo variare i prezzi relativi. Mentre il primo effetto è ineliminabile, il secondo deve essere minimizzato perché non apporta di per sé alcun vantaggio alla collettività e rappresenta un onere aggiuntivo per il soggetto colpito dall'imposta. Per la prima volta troviamo espresso in forma esplicita il concetto di eccesso di pressione, che tanta parte avrà nella teoria degli effetti delle imposte.

Alla politica economica iene riconosciuto un compito importante, che consiste principalmente nell'assecondare le energie spontanee della società e nell'impedire che queste vengano frenate da interventi controproducenti. Sia il Verri che il Beccaria avvertivano che il sistema economico sociale era in fase di cambiamento e che era perciò necessario individuare le coordinate fondamentali su cui agire per liberare le forze che dovevano promuovere, con l'aiuto della ragione, l'evoluzione della società. Questa si sviluppa perché aumentano le esigenze dei soggetti che spingono il sistema verso livelli di maggiore produzione. Per capire come doveva atteggiarsi lo stato nei confronti delle iniziative che nascevano nella società sono sufficienti frasi come queste: "Aprasi la strada ampia e libera a chiunque di esercitar la sua industria dove più vuole; lasci il legislatore che si moltiplichino i venditori in ogni classe e vedrà in breve l'emulazione e il desiderio di una vita migliore risvegliar gli ingegni, render più agili le mani del suo popolo, perfezionandosi le arti tutte, ribassarsi il livello dei prezzi, l'abbondanza dovunque guidata dalla concorrenza", ${ }^{52}$ oppure "ogni diminuzione che vorrà farsi della quantità totale del moto e nelle stabili azioni della società, sarà un passo verso la distruzione della medesima" ${ }^{53}$... "A me pare che ogni porzione di libertà che ultroneamente si tolga agli uomini sia un errore in politica" ${ }^{54} \mathrm{E}$ Beccaria, non diversamente in spirito, afferma "Nelle cose tutte, nelle quali l'interesse nostro è complicato, non è necessario di far nien-

51 P. Verri, Meditazioni sull'economia politica, in Edizione, cit., Vol. II, Tomo 2, Roma, 2007, p. 553.

52 P. Verri, Meditazioni, cit., p. 429.

53 P. Verri, Meditazioni, cit., p. 446.

54 P. Verri, Meditazioni, cit., p. 453. 
te altro che rimuovere gli ostacoli, che si oppongono allo sviluppamento di questa forza primitiva dell'animo nostro", ${ }^{5}$ e "questa universale concorrenza che aumenta il moto e l'azione, senza la quale tutto giacerebbe nel silenzio e nel vuoto". ${ }^{56}$

Da queste premesse discendono per esempio l'avversità alle privative, alla creazioni di privilegi monopolistici e alle corporazioni, contro le quali si batteranno sia Verri sia Beccaria in sede amministrativa. Analogamente venivano avversati i regimi vincolistici sui prezzi, ${ }^{57}$ si richiedeva che venissero abbattuti i dazi interni che ancora numerosi dividevano il ducato e rendevano il territorio diviso e frammentato da un punto di vista economico, restringendo le dimensioni del mercato. Coerentemente con queste impostazioni, nella questione dell'Annona e delle leggi restrittive sul commercio dei grani Verri si schierò con grande vigore contro ogni regime vincolistico e propose un piano fortemente liberista, che peraltro trovò la fiera opposizione dell'Amministrazione del Ducato e di gruppi di interesse locali più che quella di Vienna, dove invece Kaunitz vedeva quantomeno senza disfavore l'iniziativa del giovane patrizio milanese. Beccaria, sempre portato in economia a prendere posizioni equilibrate e con temperamento più cauto e pragmatico, sull'argomento ha una opinione molto più sfumata. Nel trattare della questione negli Elementi sostiene infatti che la soluzione più opportuna è la liberalizzazione del mercato dei grani; tuttavia prevede possibili eccezioni; ritiene che in una materia politicamente delicata possa essere opportuno, in presenza di circostanze particolari, limitare la libertà di contrattazione per evitare il timore o anche il sospetto di possibili carestie. ${ }^{58}$ Come funzionario Beccaria tornò ad occuparsi della questione e anche in questa veste mostrò la sua cautela, tanto che più di una volta Kaunitz, che desiderava un abbandono più rapido del regime vincolistico, espresse la sua impazienza.

In sostanza la politica economica proposta da Verri e Beccaria è di stampo essenzialmente liberale: basata, per usare un'espressione moderna, sulla libera iniziativa e sul principio del fondamentale rispetto della proprietà privata.

55 C. Beccaria, Elementi, cit., p. 79.

56 C. Beccaria, Elementi, cit., p. 304.

57 P. Verri, Meditazioni, cit., p. 449 e sgg.

58 C. Beccaria, Elementi, cit., p. 293 e sgg. 


\section{PROGRAMMI DI POLITICA ECONOMICA}

Non è difficile trovare brani di Verri e di Beccaria che contengono indicazioni sui programmi di politica economica da seguire. Prendiamo ad esempio Beccaria che scrive negli Elementi.

"Diremo che per quattro mezzi principali si aumenta il commercio di una nazione...Primo per la massima concorrenza sia de' compratori come de' venditori sian pure nazionali o esteri come si voglia, e questa si ottiene col maggior grado di libertà a tutti di fare quel commercio che più piace ...Questa concorrenza ... fa nascere i commerci utili allo stato e ... impedisce il temuto monopolio ... Secondo mezzo è il basso prezzo della mano d'opera il qual basso prezzo nasce e dalla concorrenza medesima ...onde ogni opera nel minor tempo possibile e dalle più poche mani che si può venga fatta... Il terzo consiste nella massima facilità dei trasporti ... Il quarto mezzo finalmente consiste nei bassi interessi del denaro". 59

Come si vede è un programma che si propone di favorire la concorrenza e che comprende una politica pubblica che provveda alla predisposizione di adeguate infrastrutture. Analogamente possiamo dire che Verri favorisce interventi favorevoli all'apertura, quantomeno interna, dei mercati e all'allargamento degli stessi attraverso l'abbattimento delle tariffe interne. E' noto che Verri si esprime anche a favore di interventi che favoriscono l'aumento del numero dei venditori. Come abbiamo detto gli strumenti da utilizzare sono non tanto di tipo diretto, e cioè con ordini impartiti e divieti assoluti, ma indiretto, agendo cioè attraverso incentivi e penalizzazioni.

Nell'ambito di questa limitata interferenza dello stato non si escludono interventi più diretti di politica economica. Tutti due i nostri economisti sono infatti favorevoli a opere infrastrutturali, soprattutto quelle in grado di abbassare i costi di trasporto perché abbattono le barriere che ostacolano gli scambi e il commercio. Tutto ciò che avvicina gli uomini è positivo " $\mathrm{e}$ in piccolo spazio, si anima e fermenta e perfeziona, e spande tutto all'intorno l'attività la riproduzione e la vita". Sono anche favorevoli ad interventi volti ad aumentare l'istruzione. Beccaria ad esempio propone di creare quello che noi definiremmo un

59 C. Beccaria, Elementi, cit., p. 95.

60 P. Verri, Meditazioni, cit., p. 497. 
centro di ricerca finanziato con fondi pubblici per l'innovazione e lo sviluppo della tecnologia in agricoltura.

Inoltre i nostri due autori non accettano acriticamente qualsiasi distribuzione della ricchezza e ritengono che una sua sperequata distribuzione possa provocare seri intralci al libero sviluppo della società, portando ad un utilizzo distorto e limitativo delle risorse. Verri dice "Quando le ricchezze della nazione sono costipate nelle mani di pochi (questi) cagioneranno in quello stato frequenti monopoli e frequenti carestie artificiali. Che se la sproporzione delle ricchezze sarà nelle divisioni delle terre, dico che l'agricoltura non potrà prosperarvi giammai" ${ }^{61}$ Analoga posizione è assunta da Beccaria, che afferma che "uno degli ostacoli allo sviluppo dell'agricoltura è l'essere ristrette le terre dello stato in troppe poche mani" ;22 da qui la sua avversione all'istituto e all'abuso dei fedecommessi, introdotti in gran parte dall'aristocrazia feudale. Questa posizione sembra essere dettata da un desiderio di redistribuzione non tanto mirato a rendere uguali i punti di partenza degli associati in senso meritocratico, ma diretto a risolvere o migliorare problemi di efficienza, soprattutto in senso dinamico, del sistema produttivo. E' chiaro che i due economisti milanesi vedevano il trapasso dall'antico regime alla moderna economia anche come un venir meno dei vincoli alla proprietà esistenti nel passato, che impedivano lo sfruttamento efficiente delle risorse del territorio. Le battaglie contro il fedecommesso trovano spazio anche in articoli pubblicati nel Caffè. ${ }^{63}$

Ugualmente, e questo vale soprattutto per Beccaria, l'intervento dello stato è auspicato nei casi in cui il mercato non è in grado di raggiungere soluzioni soddisfacenti. La fede liberale di Beccaria non può essere messa in dubbio ma tuttavia egli rimane ben conscio che esistono limiti alla capacità del mercato di rispondere al meglio a tutte le circostanze che si possono concretamente presentare nell'evoluzione del processo economico. In questa prospettiva non esclude la possibilità di interventi anche incisivi da parte dello stato. Particolarmente interessante la riflessione di Beccaria in tema di ottima proporzione fra le differenti colture delle terre. E' persuaso che l'ottima proporzione si raggiunga lascian-

61 P. Verri, Meditazioni, cit., p. 423.

62 C. Beccaria, Elementi, cit., p. 60.

63 Si veda ad esempio l'articolo di A. Longo, Osservazione su i fedecommessi in il Caffè. cit., pp.115-131. 
do libero il mercato di raggiungerla, ed è anche convinto che sia difficilissimo calcolare a priori quale sia l'ottima proporzione. Tuttavia, se per avventura il mercato non dovesse trovare l'ottima soluzione, suggerisce di intervenire con un sistema di tributi e sussidi molto articolato per ottenere il risultato ottimale. Beccaria inoltre individua casi di fallimento del mercato, cogliendo aspetti che successivamente saranno catalogati nelle categorie delle esternalità: casi cioè in cui il mercato lasciato a sé stesso non raggiunge le caratteristiche di ottimalità desiderate. Quando l'interesse privato non riesce a congiungersi con quello pubblico allora l'intervento correttivo si giustifica. In primo luogo quando esistono condizioni di asimmetrie informative, come Beccaria individuò lucidamente molto prima che questa categoria analitica venisse poi elaborata dagli economisti: "Dunque la disciplina delle arti non deve essere coattiva e legislatrice, se non dove si prevegga che non mai o troppo tardi l'interesse privato giungerà ad unirsi col pubblico e dove cioè la scoperta di frodi è lenta ed il guadagno che apportano è presente e considerevole". ${ }^{64}$ In questo caso sono opportuni controlli e certificazioni.

Vi sono casi poi in cui (ad esempio i boschi) Beccaria è in favore di una regolazione del settore, dimostrando in maniera del tutto corretta come gli orizzonti temporali normali dei privati possano portare a risultati non ottimali quando i rendimenti sono realizzabili compiutamente solo nel lungo periodo. ${ }^{65}$

\section{POLITICA TRIBUTARIA}

Verri affronta in modo sistematico il tema tributario sia nelle Meditazioni sia in numerosi scritti e importanti consulte; ${ }^{66}$ manca un'analoga trattazione organica in Beccaria in quanto la parte del corso prevista nell'originario piano delle lezioni dedicata ai tributi probabilmente non venne mai svolta. Abbiamo quindi solo dei riferimenti sporadici

64 C. Beccaria, Elementi, cit., p. 331.

65 C. Beccaria, Elementi, cit., p. 316 e sgg.

66 Il tema dei tributi è trattato da Verri in numerosi scritti; tra gli altri Meditazioni cit., Voto sul nuovo piano toccante le arti e mestieri sull'università, Piano per la regia amministrazione delle finanze. Gli ultimi due scritti si trovano in P. Verri, in Edizione, cit., Vol. II, tomo I pp.753-766 e 780-800. 
negli Elementi e delle trattazioni su punti specifici del sistema tributario (ad esempio qualche consulta sulla tassa mercimoniale).

Verri definisce il " tributo parte la più importante ed irritabile del corpo politico, e tenta di illustrare "perché le leggi tributarie trovano sempre un niso continuo nella nazione per opporvisi". ${ }^{67} \mathrm{La}$ spiegazione ha più facce: da un lato vi è la miopia del contribuente che, mentre avverte l'immediata sottrazione del bene privato, non coglie appieno i benefici lontani che vengono dall'essere assicurati da un'eventuale violenza. In secondo luogo l'idea della proprietà privata è molto più radicata nell'animo dell'uomo di quanto non sia l'idea generale dell'organizzazione dello stato. Tuttavia, riecheggiando Montesquieu, per Verri questo diverso sentire dipende in larga misura dal comportamento della classe direttrice: laddove per lungo tempo la legge si è rivelata saggia e finalizzata al bene comune, il rifiuto a pagare viene sentito come un'azione riprovevole. Il costume, la storia, i valori di una società finiscono per incidere sul concreto funzionamento dei sistemi tributari. ${ }^{68}$

Il tributo trova la sua giustificazione di fondo nell'assolvere a due principali compiti. Il primo concerne il pagamento della classe chiamata dal Verri direttrice, indispensabile perché chiamata a fornire il quadro giuridico istituzionale senza il quale viene a mancare la possibilità stessa di condurre agevolmente l'attività di riproduzione; si devono cioè mantenere ministri, funzionari, magistrati, soldati dediti a questi compiti: "Il tributo è perciò una porzione della proprietà che ciascuno depone all'erario pubblico al fine di godere con sicurezza la proprietà che gli rimane". ${ }^{99}$ Curiosamente questa frase di Verri corrisponde a quella di Beccaria scritta peraltro in un diverso contesto: "Essi ne sacrificarono una parte (di libertà) per godere per il restate con sicurezza e tranquillità". ${ }^{70} \mathrm{Si}$ anticipano le tesi dei sostenitori del principio del beneficio, tra i quali Adam Smith che sostiene che il carico dell'imposta per il singolo dovrebbe corrispondere al vantaggio che questi riceve dalla spesa pubblica. Verri approva il fatto che il tributo, in ultima analisi, sia sopportato dai possessori, perché esso serve appunto in primo luogo a difendere la proprietà di questa classe.

67 P. Verri, Meditazioni, cit., p. 518.

68 P. Verri, Meditazioni, cit., p. 519.

69 P. Verri, Meditazioni, cit., p. 517.

70 C. Beccaria, Dei delitti, cit., $\$ 1$. 
Stando l'inevitabilità delle imposte si pone tuttavia il problema di avere un sistema tributario funzionale al buon andamento dell'economia Verri riconosce che il sistema tributario ha effetti sull'annua riproduzione, diminuendola ma anche accrescendola a seconda di come viene regolato. Saggiamente collocato, per esempio, può aiutare le manifatture o l'agricoltura, contribuendo allo sviluppo del paese attraverso dazi all'importazione o all'esportazione. ${ }^{71}$ Sull'uso delle tariffe a fini di incentivi all'attività economica Beccaria concorda pienamente con Verri. ${ }^{72}$ Ambedue quindi appartengono a quel filone di economisti italiani impegnati a sviluppare la teoria dell'imposta ottima, pensata per favorire lo sviluppo economico, distinguendosi in ciò dalla scuola anglosassone impegnata ad individuare l'imposta neutrale diretta a lasciar inalterata la posizione relativa dei soggetti.

I tributi devono quindi essere regolati in modo da raggiungere le finalità volute: protezione per la proprietà (sostentamento della classe di governo), aiuto o quantomeno non intralcio alla vita economica, finanziamento di opere pubbliche.

Il sistema fiscale può provocare però anche notevoli danni, vuoi per una pressione fiscale troppo elevata vuoi per il modo in cui i tributi vengono imposti. Nel primo caso, cioè quando la quantità del tributo eccede le forze della nazione e non è proporzionato alla ricchezza del paese, l'intralcio allo sviluppo è un conseguenza sicura. La spesa pubblica che supera i limiti di sopportabilità del sistema produttivo è principalmente, nell'ottica di quel periodo, dovuta a interventi di tipo bellico o a sprechi del sovrano. In ambedue i casi le risorse sottratte al sistema hanno impieghi improduttivi e lasciano risorse insufficienti a garantire lo sviluppo. ${ }^{73}$

Nel secondo caso i danni sono provocati da una distribuzione "viziosa": questo avviene se non vengono rispettate regole di buona gestione del sistema tributario. Verri ne individua cinque ricorrenti nella letteratura del periodo; esse in larga misura anticipano i famosi criteri di Adam Smith per un buon sistema tributario.

Il primo canone richiede che il tributo non piombi immediatamente sopra la classe dei cittadini più deboli. Per capire il perché di

71 P. Verri, Meditazioni, cit., p. 545.

72 C. Beccaria, Elementi, cit., p. 350.

73 P. Verri, Meditazioni, cit., p. 520. 
questa affermazione bisogna ricordare la teoria dell'incidenza delle imposte di Verri; secondo l'economista milanese infatti solo i consumatori possessori sono in definitiva colpiti dall'imposta, chiunque sia il contribuente di diritto. Qual è allora la ragione per cui non vuole che si colpisca in prima istanza la classe più debole? Le spiegazioni fornite mostrano ancora una volta la sensibilità del Verri a cogliere i fatti economici in generale e quelli tributari in particolare, tenendo conto della rottura anche temporanea degli equilibri economici.

"Sembra dunque, a primo aspetto perché il tributo tenda a conguagliarsi sulle consumazioni che arbitrario sia lo scegliere anzi una classe che l'altra del popolo; ma ciò non è perché questo conguaglio e questa suddivisione del tributo è sempre uno stato di guerra fra ceto e ceto di uomini. Quando il possessore o il cittadino che ha fondi debbono anticipare il tributo, la suddivisione sul minuto popolo si fà immediatamente $\mathrm{e}$ con poco ostacolo perché egli è potente che richiede ragione al debole; ma quando il tributo cada immediatamente sulla classe del più debole, la suddivisione si farà ma con quella lentezza e quegli ostacoli che debbono nascere quando il debole e il povero cerca ragione del ricco e dal potente. Questi intervalli fra l'impulso e la quiete sono le crisi più importanti per gli stati e son ben da osservarsi in ogni cambiamento di tributo". ${ }^{74}$

Analoghe considerazioni vengono fatte dal Verri nel discutere l'opportunità di ricorrere alla capitazione: essa viene rifiutata non tanto sulla base di ragioni equitative di lungo periodo (perché nella visione dell'incidenza dell'imposta nel lungo periodo i cittadini "poveri" scaricheranno l'onere del tributo sui possessori consumatori), ma perché nel breve periodo questo tipo di imposta ha contro di sé il tempo del "conguaglio", cioè lo spazio in cui il povero fa la guerra al ricco.

Il secondo canone prevede che sia minimizzato il costo di esazione (si sente qui la battaglia che Verri condusse contro la Ferma Generale, formula di riscossione che, assegnata ai privati, comportava costi aggiuntivi e grandi profitti per i fermieri.)

Il terzo richiama uno dei punti ritenuti molto importanti da Verri, cioè la certezza del cittadino: "Ch'egli abbia per norma leggi chiare, precise, inviolabili, da osservarsi imparzialmente verso di qualunque contribuente". ${ }^{75}$

74 P. Verri, Meditazioni, cit., p. 524.

75 P. Verri, Meditazioni, cit., p. 528. 
Il quarto riguarda gli ostacoli che le imposte possano frapporre agli scambi interni al paese: quindi abolizione dei dazi interni e delle imposte sui contratti. Questo punto, dazi interni, era allora importante per il Milanese in quanto il paese era diviso in sei circoscrizioni tributarie e gli scambi commerciali erano parecchio distorti da queste barriere interne. Si auspicano norme chiare, semplici, precise e inviolabili da far osservare imparzialmente a tutti contribuenti. Si ritiene inoltre essenziale, come del resto abbiamo già accennato, abolire tutti i dazi interni per non intralciare la vita economica. Sulla questione più volte Verri interverrà, anche se non con molto successo. La riforma da lui proposta sarà accettata solo nel 1786.

L'ultimo principio afferma che "il tributo non deve mai seguire l'accrescimento dell'industria". Questo canone rispecchia in modo più caratteristico le idee Verriane: "a misura che l'uomo cerca di migliorar la sua sorte coll'attività dell'industria, gli cadrà proporzionalmente sul capo un sopraccarico di tassa sul tributo, questo tributo sarà diametralmente opposto a' progressi dell'industria e tenderà direttamente ad impedire l'avanzamento dell'annua riproduzione".$^{76}$ Sempre con l'intento di non intralciare lo sviluppo del sistema il Verri non si limita ad appoggiare il sistema catastale ma propone che per i primi anni le nuove attività, siano esse nel settore agricolo o in quello manifatturiero, vengano esentate da imposte.

La proposta di riforma del sistema tributario avanzata nelle Meditazioni, in sede di riflessione teorica, e ripresa nella consulta Piano per la regia amministrazione delle finanze come progetto formulato all'interno della Amministrazione e ribadito ancora nella nota Pensieri sullo stato politico del milanese, riflette molto bene il senso dei cinque canoni che aveva indicato nelle Meditazioni.

Le imposte previste sono solo due: l'una che colpisce i terreni mediante il catasto, l'altra l'insieme dei dazi all'importazione e all'esportazioni. Infatti viene rispettato il principio della semplicità del sistema e delle leggi, vengono aboliti i dazi interni, si evita che l'imposta cada immediatamente sul ceto più debole e vengono introdotte imposte che sono incentivanti per "l'annua riproduzione". La prima imposta, quella catastale, ha una evidente funzione premiale: l'imposta, proprio perché calcolata sul reddito normale e non su quello effettivo, non

76 P. Verri, Meditazioni, cit., p. 528. 
aumenta al crescere del reddito; inoltre, essendo imposta a somma fissa, non produce eccesso di pressione.

Nell'idea di Verri i dazi dovrebbero essere non solo un mezzo per aumentare gli introiti dello stato ma dovrebbero anche essere utilizzati per una sorta di politica industriale. Su questo punto le proposte di Verri sono totalmente condivise da Beccaria. ${ }^{77}$ Le imposte all'esportazione dovrebbero colpire la materia prima in modo da favorire il suo utilizzo interno e accrescere l'industria manifatturiera interna, mentre i dazi sulle importazioni dovrebbero favorire le produzioni nazionali.. Per i due economisti quindi in questo settore si dovrebbe abbandonare lo schema liberista. Due osservazioni in proposito: questa loro posizione, che si può assimilare a quella tardo mercantilistica, in realtà è dettata da quella corrente che mirava attraverso lo scambio con l'estero ad aumentare il livello dell'occupazione in quanto non veniva ipotizzata l'esistenza della piena occupazione. In Verri inoltre troviamo qualche scrupolo per l'abbandono della posizione liberista, tanto che si domanda: " $E$ ' stato proposto il quesito se qualora tutte le nazioni si accordassero ad abolire i tributi sulle merci cosicché liberamente e senza verun carico ogni merce potesse entrare o uscire in uno stato se, dico questa operazione sarebbe universalmente giovevole, ovvero quali effetti produrrebbe? Se questo accordo fra le potenze d'Europa fosse sperabile, è molto facile il prevedere quali ne sarebbero le conseguenze, cioè le medesime che nascono in uno stato togliendo i tributi sull'interna circolazione. Si accosterebbero le nazioni fra di loro, si moltiplicherebbero i contratti; l'industria generalmente e l'annua riproduzione si rianimerebbero per tutta l'Europa; gli uomini godrebbero di comodi maggiori, ma la potenza degli stati, cioè la relazione che ha uno stato con l'altro rimarrebbe la medesima". ${ }^{78}$

E' stato osservato che l'idea di Verri di applicare nel settore delle finanze l'intervento diretto viene contraddetto dall'uso dei dazi doganali in quanto intervento indiretto. ${ }^{79}$ L'osservazione è certamente giusta ma è opportuno precisare che per Verri le tariffe sono anche strumento di politica industriale, utilizzato per incentivare l'attività economica: quantomeno si tratta di un intervento con una pluralità di finalità.

77 C. Beccaria, Elementi, cit., Vol. XII, p. 311.

78 P. Verri, Meditazioni, cit., p. 544.

79 P. Porta, in P. Verri, Edizione, cit., vol. II, tomo II, p. 554, nota 118. 
Verri si sofferma inoltre sul modo di introdurre le riforme nel sistema tributario: bisogna procedere cautamente perché "il tributo ...non può mai essere scomposto con violenza e con impeto" ${ }^{80}$

Aggiunge anche con tipico fiducioso atteggiamento illuminista: "Promuovere dunque $i$ lumi e la curiosità nelle materie di finanza e di commercio sarà sempre la preparazione migliore di tutta e per cominciar le riforme" ${ }^{81}$

Nelle Meditazioni, alla fine della parte dedicata ai tributi, Verri in polemica col fratello Alessandro e con una corrente di pensiero allora in voga, afferma che in ogni circostanza il tributo, ancorché necessario, ha sempre anche conseguenze negative.$^{82}$ Non aderisce quindi alla tesi secondo la quale il tributo, abbassando il reddito disponibile, potesse costituire un incentivo per una maggior offerta di lavoro.

\section{POLITICA MONETARIA}

Anche nel caso della politica monetaria appare chiaro l'importanza che i due autori attribuiscono al modo di operare degli agenti economici e al concreto funzionamento del mercato. Come è noto Beccaria e Verri entrano nel dibattito sul disordine delle monete esistente allora nello stato di Milano chiarendo i possibili rimedi fin dal 1762. Il primo con un saggio dal titolo Del disordine e dei rimedi delle monete nello stato di Milano nell'anno 1762, il secondo con il dialogo Fronimo e Simplicio scritto a sostegno dell'allora amico che era stato criticato. Il saggio di Beccaria (è il suo primo lavoro su temi economici) apre il dibattito sostenendo, con il pieno accordo e sostegno di Verri, che il disordine esistente derivava da una sbagliata politica che determinava $\mathrm{i}$ cambi delle monete allora circolanti nel Milanese senza tener conto delle leggi economiche. Il dato di partenza era l'esistenza di rapporti di cambio ufficiali fissati dalle Grida per le monete che circolavano nel paese (si tenga presente che in quel periodo nel milanese avevano corso ufficiale ben 51 monete, 22 d'oro e 29 d'argento) non corrispondenti ai valori con i quali le monete venivano effettivamente scambiate. Se le

80 P. Verri, Meditazioni, cit., p. 546.

81 P. Verri, Meditazioni, cit., p. 548.

82 P. Verri, Meditazioni, cit., p. $₫$ XXXVI. 
valutazioni di mercato divergono da quelle ufficiali si verificherà una rarefazione delle monete pregiate e un eccesso di monete a bassa lega, che possiedono un valore intrinseco inferiore a quello nominale. Le "monete buone", quelle che sono sottovalutate dalla tariffa ufficiale rispetto al valore del metallo in esse contenuto, saranno oggetto di incetta, esportazione e riconiazione. Fenomeno contrario per le "monete cattive", che invece tendono ad invadere il mercato. Questa differenza di valutazione non dipende secondo Beccaria da un comportamento perverso dei cittadini ma piuttosto da una precisa ragione economica. Per avere coincidenza tra valore di mercato e quotazione ufficiale, e quindi un sistema ben regolato, è necessario che la stessa quantità di oro fino presente in diverse monete sia valutato sempre allo stesso modo, e quindi con lo stesso valore in lire. Uguale regola deve valere per qualsiasi altro metallo (argento, rame, etc.): “....ovvero che vi sia una costante equazione fra il valor fisico e il valor numerario". ${ }^{83}$

Secondo requisito per un sistema ben regolato è che il rapporto tra il valore dell'oro e quello dell'argento venga applicato uniformemente in ciascun tipo di moneta; secondo Beccaria il rapporto tra il valore dell'oro e quello dell'argento doveva corrispondere al valore medio europeo. Sul punto il parere di Verri, come risulta dalla sua consulta del 72, divergeva da quello di Beccaria: egli infatti riteneva che il rapporta tra il valore dell'oro e quello dell'argento dovesse essere calcolato tenendo soprattutto conto del rapporto esistente negli stati limitrofi, e soprattutto negli stati con cui si avevano i maggiori rapporti commerciali.

Poiché però il rapporto tra i valori di mercato dei metalli non è fisso nel tempo (quale che sia il punto di riferimento preso) ma è determinato dall'andamento complessivo dalla domanda e dall'offerta, e cioè dal diverso andamento del commercio e da nuove scoperte di giacimenti d'oro o d'argento, Beccaria ritiene necessaria la creazione di un ministro dedicato non tanto a condurre una complessiva politica monetaria quanto a gestire principalmente i corretti rapporti fra le monete.

Infine veniva discussa l'opportunità di coniare monete d'argento del Ducato. Verri affronta la questione sia nel dialogo Fronimo e Simplicio sia nella consulta del 1772. La sua opinione rimane immutata: in generale si può battere moneta profittevolmente se si hanno miniere

83 C. Beccaria, Del disordine, cit., p. 478. 
di oro o di argento; oppure se il paese, per commercio, possiede grandi quantità dei due metalli preziosi o è molto grande; o se vi sono particolari rapporti commerciali con un'altra nazione che acquista la moneta nazionale a un prezzo che compensi le spese di monetazione. In caso contrario non bisogna procedere a battere moneta, perché ci si accollano inutilmente i costi di produzione della zecca. Passando poi al caso milanese, grande peso assegna alla circostanza che, data la struttura dell'economia, la maggior parte dei contratti viene stipulati in monete estere; in uno stato piccolo come quello di Milano "che ha tutte le sue relazioni principali co' finitimi non può circoscrivesi il giro dei pagamenti, in modo che il danaro interno non esca e l'esterno non entri con un perenne moto essendo la sfera dei nostri contratti assai più vasta di quella de' confini" ${ }^{84}$

Ci sembra di dover concordare con Porta e Scazzieri che vedono in questa tesi di Verri una critica all'associazione tra standard monetari e sovranità nazionale e un sostegno alla idea "...che l'obiettivo della politica monetaria dovrebbe essere quello di avere buona moneta e di assicurare il corretto valore senza guardare ai simboli che essa reca". ${ }^{8}$

Beccaria, che per primo nel Del Disordine si era espresso per l'inopportunità di una coniazione locale, cambia invece opinione nella consulta da lui redatta nel 72 . Le spiegazioni fornite non sembrano essere particolarmente pregnanti e comunque non fanno alcun riferimento alla sua precedente presa di posizione; si può quindi avere il sospetto che questo cambiamento si debba soprattutto al fatto che Beccaria non volesse contrastare le preferenze espresse da Vienna. ${ }^{86}$

Il taglio internazionale e la fiducia nell'operare dei meccanismi di mercato si ritrovano anche nella risposta che Verri fornisce al quesito posto da Vienna su quali monete estere debbano aver corso nel ducato milanese; secondo Verri tutte le monete di qualunque metallo (rame, bronzo, argento, oro) devono essere accettate, purché si abbia garanzia della loro bontà come peso e qualità. Vanno accettate e valutate secon-

84 P. Verri, Consulta su la riforma delle monete dello stato di Milano in (a cura di) A. Quadrio Curzio, R. Scazzieri, Sul disordine delle monete a Milano nel Settecento, Milano, 1986, p.127.

85 P. Porta, R. Scazzieri, Il contributo di Pietro Verri alla teoria economica, in C. Capra, a cura di, Pietro Verri e il suo tempo, Bologna, 1999, p. 842, nota 41.

86 Si veda C. Capra, Gli intellettuali, cit. 
do il loro intrinseco valore anche per i pagamenti dei tributi. Questo sarebbe di grande vantaggio per lo Stato di Milano perché faciliterebbe la circolazione monetaria e libererebbe "il popolo dalla continua angustia di dover comprare le monete per ogni pagamento di tributo, o essere soggetto ad arbitrarie usure per il conguaglio della rifiutata dalla legge con quelle dalla legge ammesse" ${ }^{87}$

\section{CONCLUSIONI}

Le tesi di Beccaria e di Verri sulla politica economica hanno il loro fondamento nel desiderio di garantire all'individuo un spazio di libertà e di autonomia. Questo si può ottenere in un quadro giuridico adatto al raggiungimento dell'obiettivo: quindi l'accento sulla necessità di avere leggi generali che diano certezza e sicurezza e che consentano una notevole scelta nelle iniziative a carattere economico. Coerentemente pensano ad un sistema costituzionale basato sulla divisione dei poteri, in particolare tra il legislativo e il giudiziario, che limiti implicitamente l'intervento dello stato. Hanno ambedue una visione sostanzialmente liberale, dove posto preminente ha la libertà "civile"(individuale), che solo nel Verri maturo tende anche a comprendere le libertà politiche.

Per quanto riguarda lo specifico punto della politica economica, nell'ambito di un programma che mette in grande rilievo l'obiettivo dello sviluppo, entrambi danno notevole importanza all'iniziativa economica individuale: compito della legislazione è permettere alle forze spontanee della società di operare al meglio. Gli interventi dello stato sono limitati ma non per questo meno importanti: perché devono fornire il quadro giuridico e predisporre le infrastrutture, soprattutto quelle di trasporto, necessarie ad una vivace attività economica Tutti e due sono anche attenti a individuare $i$ casi in cui meccanismi di mercato non funzionano al meglio rendendo necessario l'intervento correttivo dello stato. Il modello di politica economica adottato da entrambi è sostanzialmente comune; vi sono tuttavia differenze tra i due, quanto meno di accento. Beccaria in generale è molto più prudente nell'accettare gli esiti del mercato (valga per tutti l'esempio dell'Annona).

Su un punto si differenziano in modo molto netto da quella che

87 P. Verri, Consulta, cit., p.130. 
sarà la posizione degli economisti liberali dell'800: auspicano infatti nel commercio con l'estero l'utilizzo di tariffe per sviluppare al meglio le attività produttive. Ferrara ${ }^{88}$ criticò vigorosamente $\mathrm{i}$ due economisti milanesi per questo loro indirizzo. Ci sembra però di poter concordare con Schumpeter che le osservazioni dell'economista siciliano sono troppo severe: non tengono conto che le proposte dei due milanesi rappresentavano un moderato protezionismo rispetto alle dottrine precedenti e che comunque avrebbero potuto rappresentare "a better starting point for further research than was the narrow dogmatism of the freetrade doctrine that replaced (them)" ${ }^{89}$

88 F. Ferrara, Prefazione, in Biblioteca dell' economista, $1^{\text {a }}$ serie, $3^{\circ}$ vol., Trattati italiani del XVIII secolo: Genovesi, Verri, Beccaria, Filangieri, Ortes, Torino, 1852.

89 J. Schumpeter, History, cit., p. 375. 